\title{
Dual Extraction of Photogenerated Electrons and Holes from a Ferroelectric $\mathrm{Sr}_{0.5} \mathrm{Ba}_{0.5} \mathrm{Nb}_{2} \mathrm{O}_{6}$ Semiconductor
}

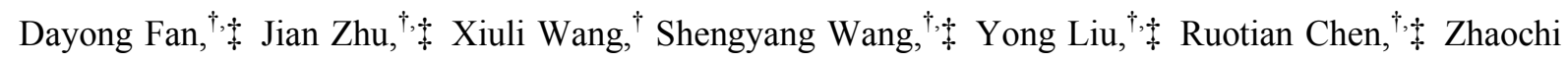
Feng, ${ }^{\dagger}$ Fengtao Fan, ${ }^{, \dagger}$ Can $\mathrm{Li}^{*}, \dagger$

$\dagger$ State Key Laboratory of Catalysis, Dalian Institute of Chemical Physics, Chinese Academy of Sciences; Dalian National Laboratory for Clean Energy, Dalian 116023, China

$\$$ Graduate University of Chinese Academy of Sciences, Beijing 100049, China.

Corresponding Authors: E-mail: canli@dicp.ac.cn.

E-mail*: ftfan@dicp.ac.cn. 
UV-visible absorption spectroscopy measurement

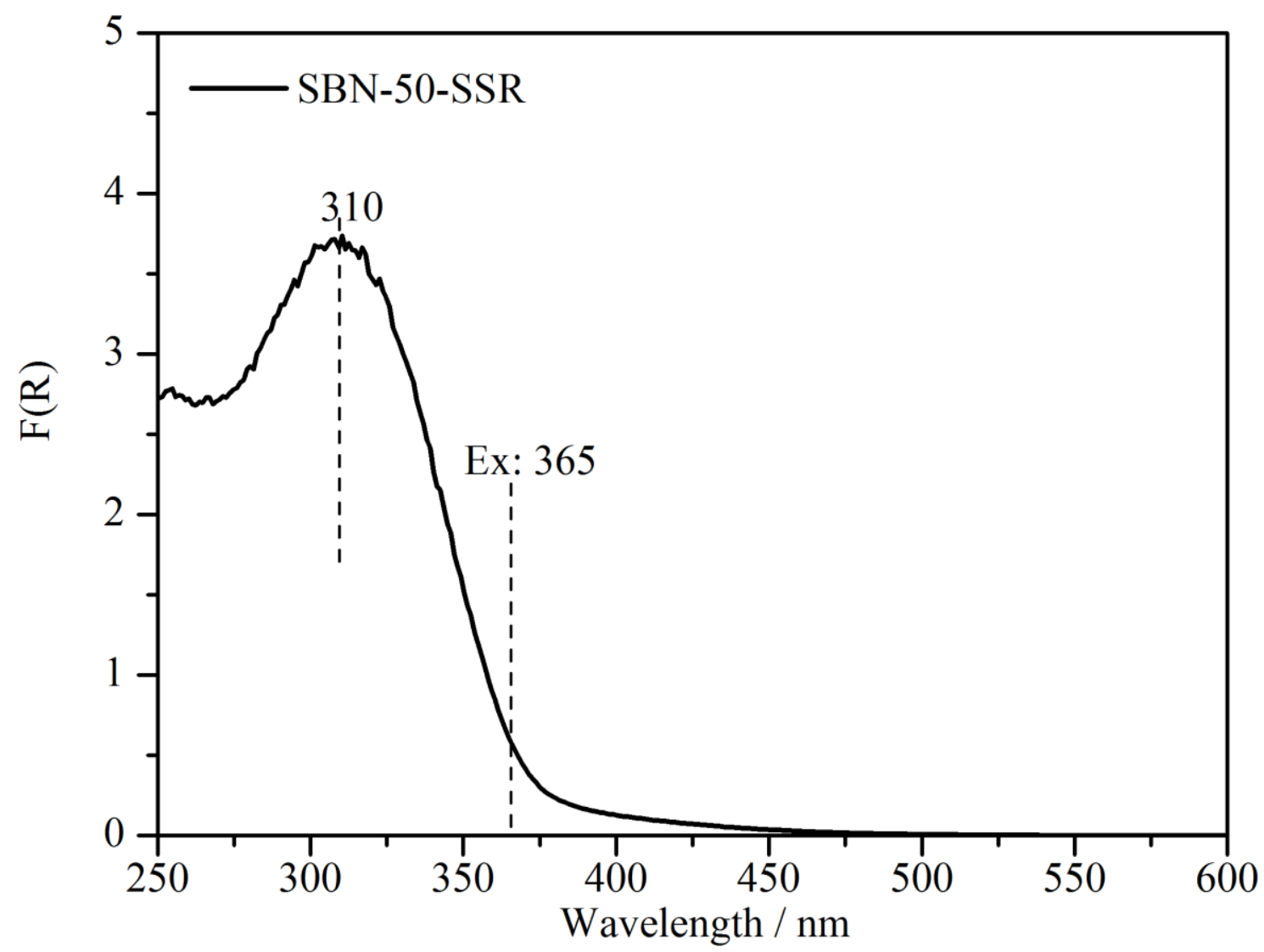

Fig. S1 Ultraviolet-visible diffuse reflectance spectra of SBN-50-SSR powder taken $F(R)$ as $Y$ axis, where $F(R)$ is the Kubelka-Munk function of the diffuse reflectance calculated by $F(R)=(1-R)^{2} / 2 R$ 


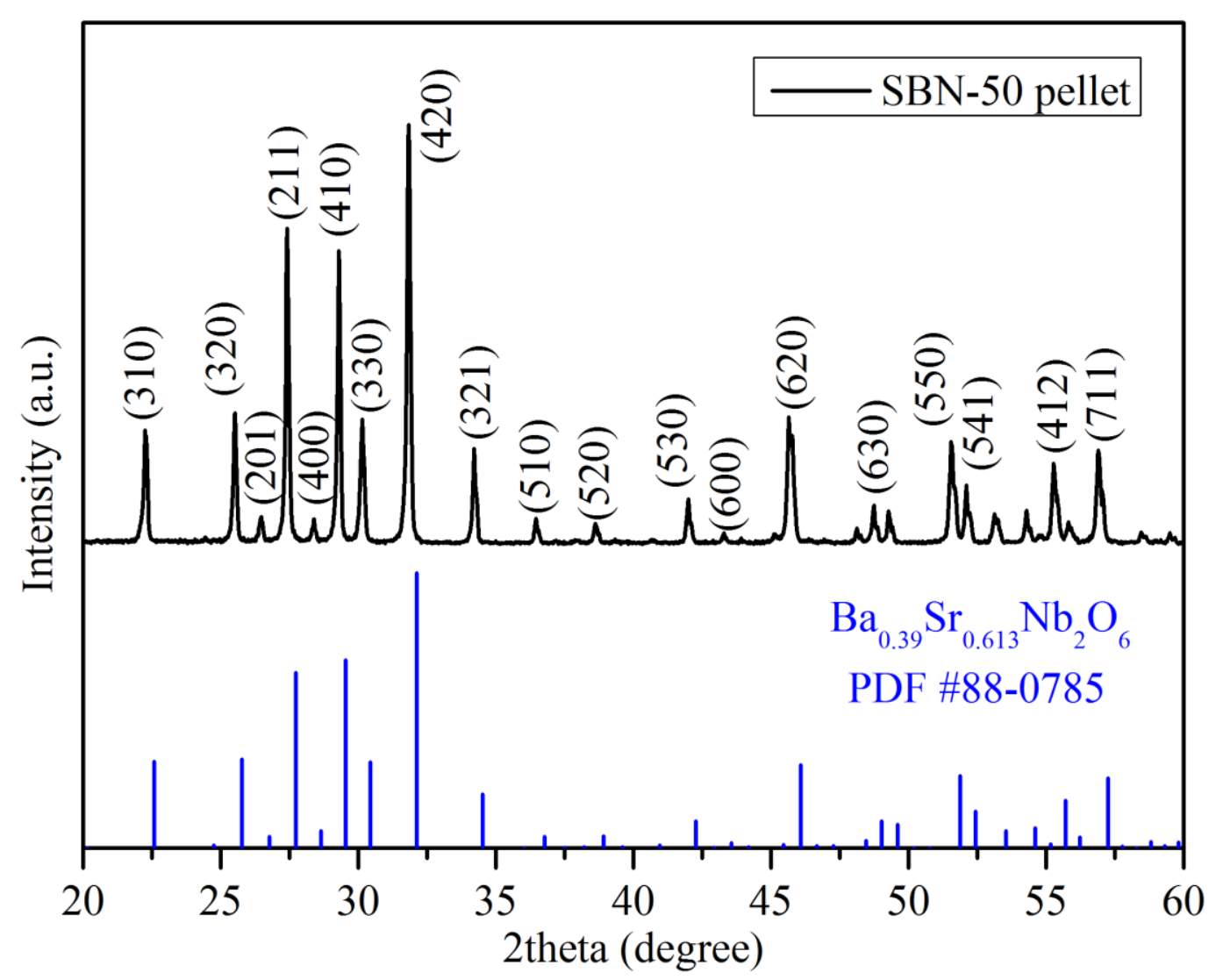

Fig. S2 X-ray powder diffraction pattern of sintered SBN-50 pellet, which matches well with the standard JCPDS \#88-0785 tetragonal tungsten bronze SBN structure, no second phase appears.

Table $\mathrm{S} 1$. The atom ratios $(\mathrm{Sr}, \mathrm{Ba})$ relative to $\mathrm{Nb}$ in the sintered $\mathrm{SBN}-50$ pellet, was measured by ICP-OES (Inductively Coupled Plasma Optical Emission Spectrometry) method:

\begin{tabular}{|c|c|c|c|}
\hline & $\mathrm{Sr}$ & $\mathrm{Ba}$ & $\mathrm{Nb}$ \\
\hline No. $^{\mathrm{a}}$ & $0.4837 \pm 0.0286$ & $0.5418 \pm 0.0892$ & 2 \\
\hline No. $2^{\mathrm{a}}$ & $0.4847 \pm 0.0083$ & $0.5349 \pm 0.0015$ & 2 \\
\hline
\end{tabular}

${ }^{a}$ Before microwave digestion, the ceramic was crushed and ground. Two times measurements are performed. 

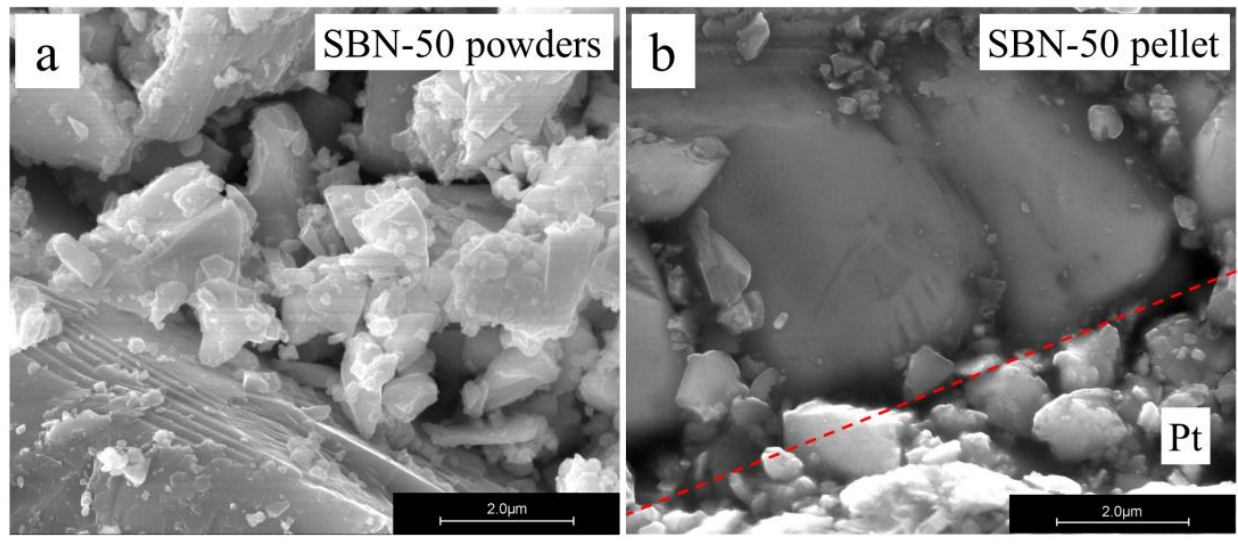

Figure S3. SEM photograghs of SBN-50 powders (a) and SBN-50/Pt interface (b)

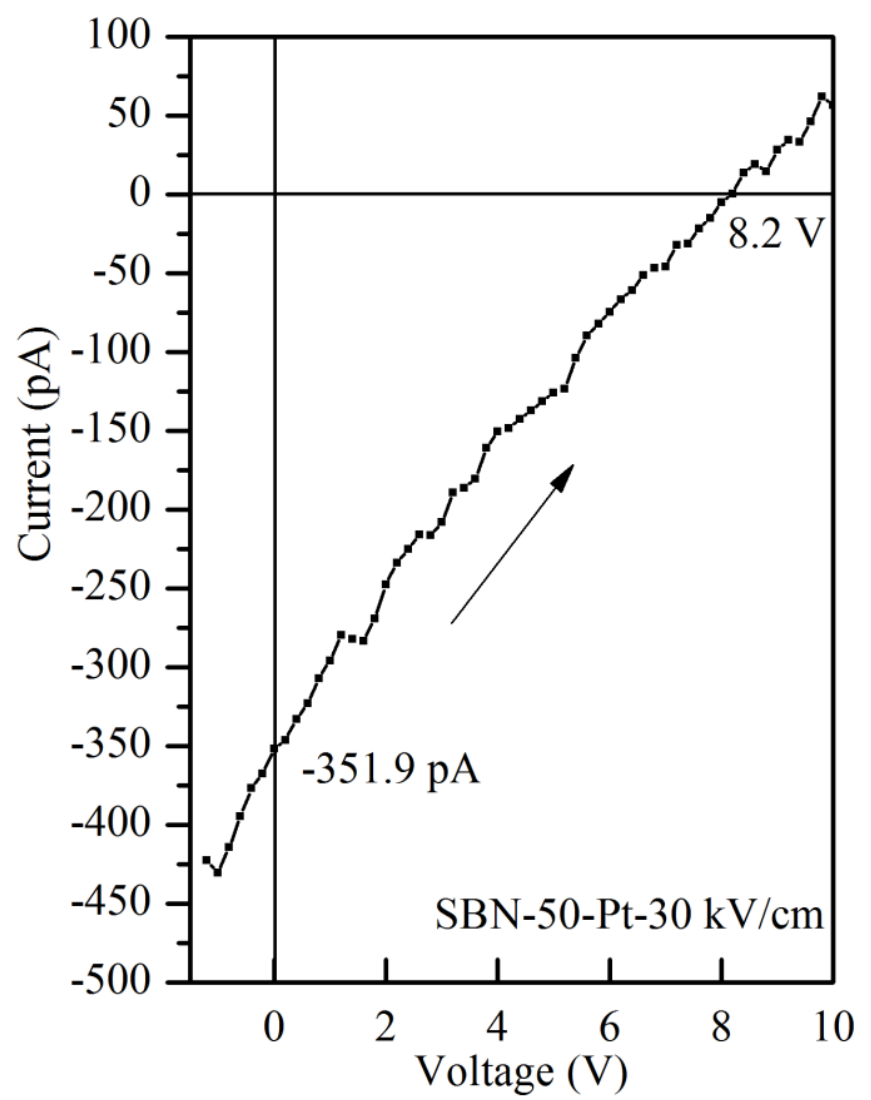

Fig. S4 I-V curves under $365 \mathrm{~nm}$ LED illumination: the poled SBN-50 with symmetrical Pt electrodes. The curve corresponding to the first time scan after poling with $E=30 \mathrm{kV} / \mathrm{cm}$ for $30 \mathrm{mins}$, which shows $\mathrm{V}_{\mathrm{oc}}$ was as high as $8.2 \mathrm{~V}$ and $\mathrm{I}_{\mathrm{sc}}$ was $-351.9 \mathrm{pA}$. The arrow in the figure indicate the scanning direction. 


\section{Photoelectric test method reliablity and APV effect stability of SBN}

For the large impedance of the SBN-50 pellets, it is hard to measure the DC current correctly. The high RC time constant and the high additional displacement current always induce large deviation from the real DC current. Here, using the voltage sweep method, data acquisition with long holding time (10s) for each point was adopted to avoid the disturbance of the displacement current. Besides, the long holding time (10s) ensures the collected data are after the dielectric relaxation of the capacitor. This is demonstrated by overlap of the forward sweep and backward sweep(see Fig. S5). Forward scan: from negative voltage to positive voltage.
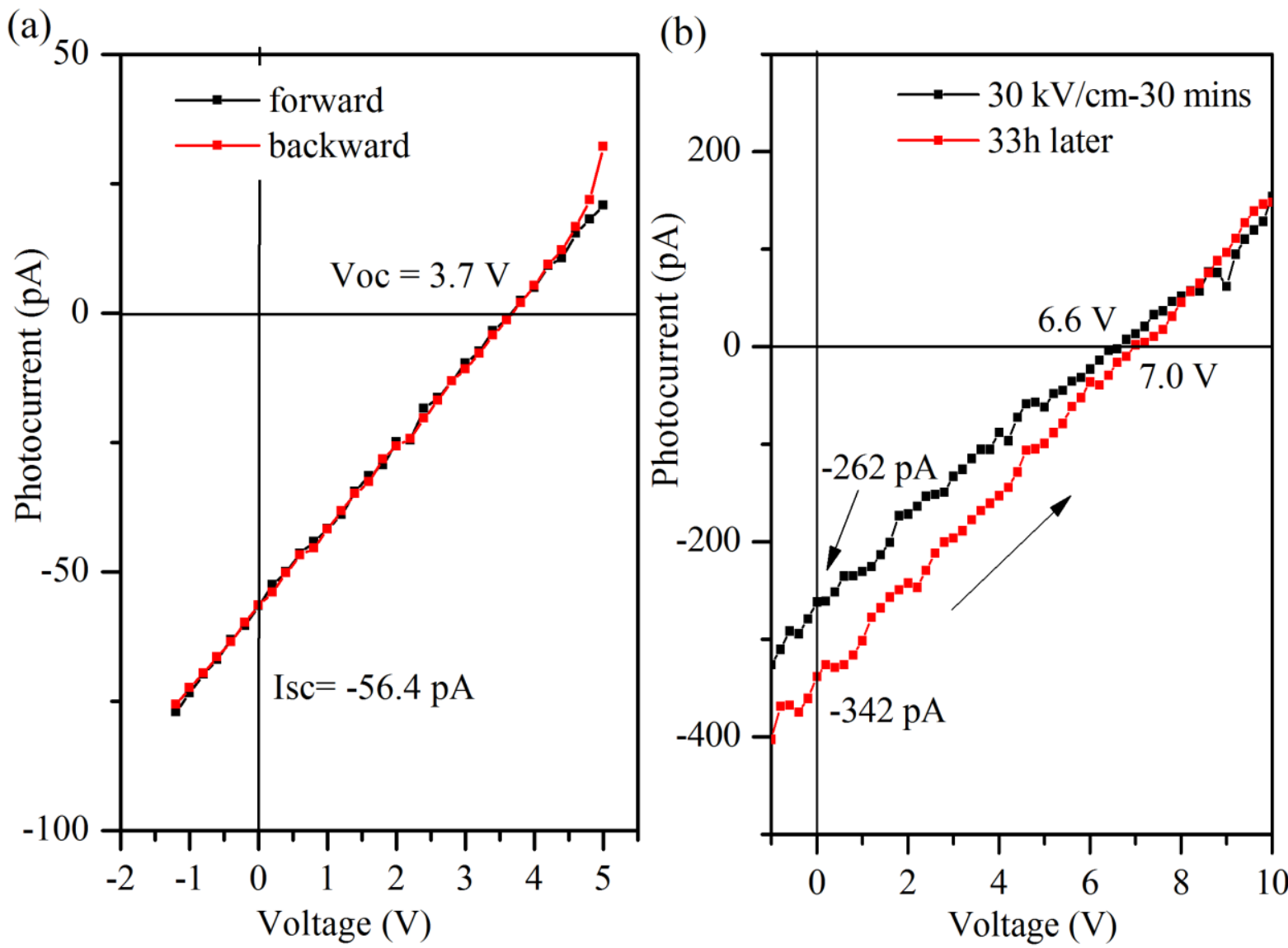

Fig. S5 (a) I-V curves under 365nm LED illumination: SBN-50-Pt sample poled with $10 \mathrm{kV} / \mathrm{cm}$ for 30 mins. The forward sweep result (black) coincides with the backward one (red). (b) The PV output of SBN-50-Pt poled with $\mathrm{E}=30 \mathrm{kV} / \mathrm{cm}$ remained stable even after the sample had been perserved under ambient condition for 33 hours. 
(a)

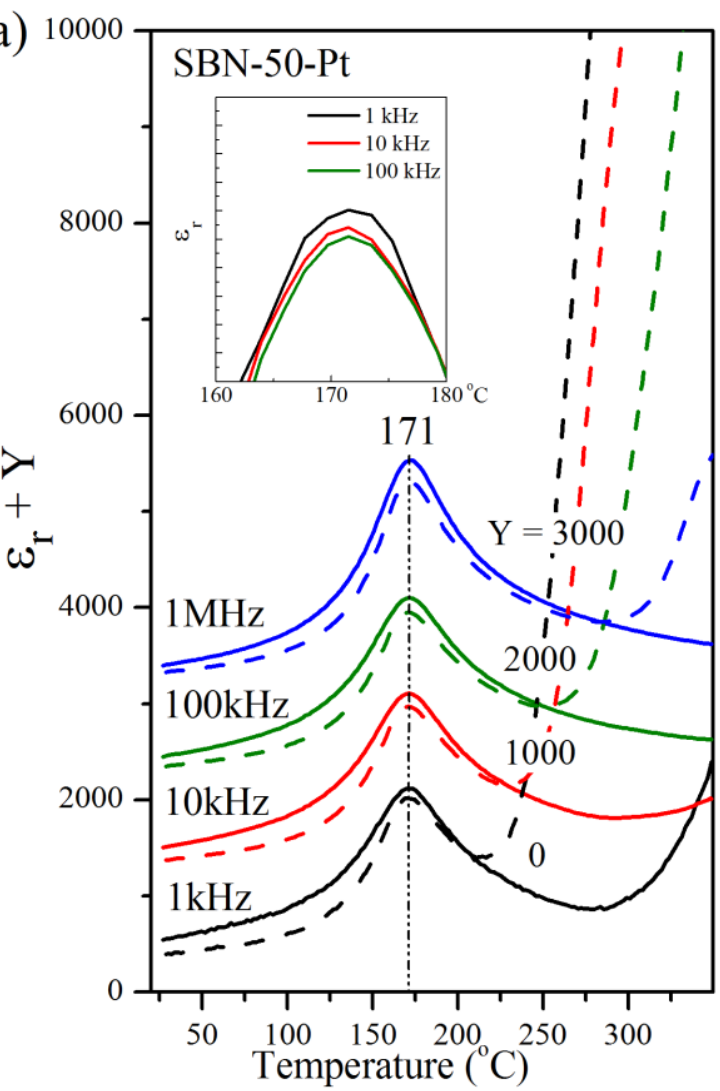

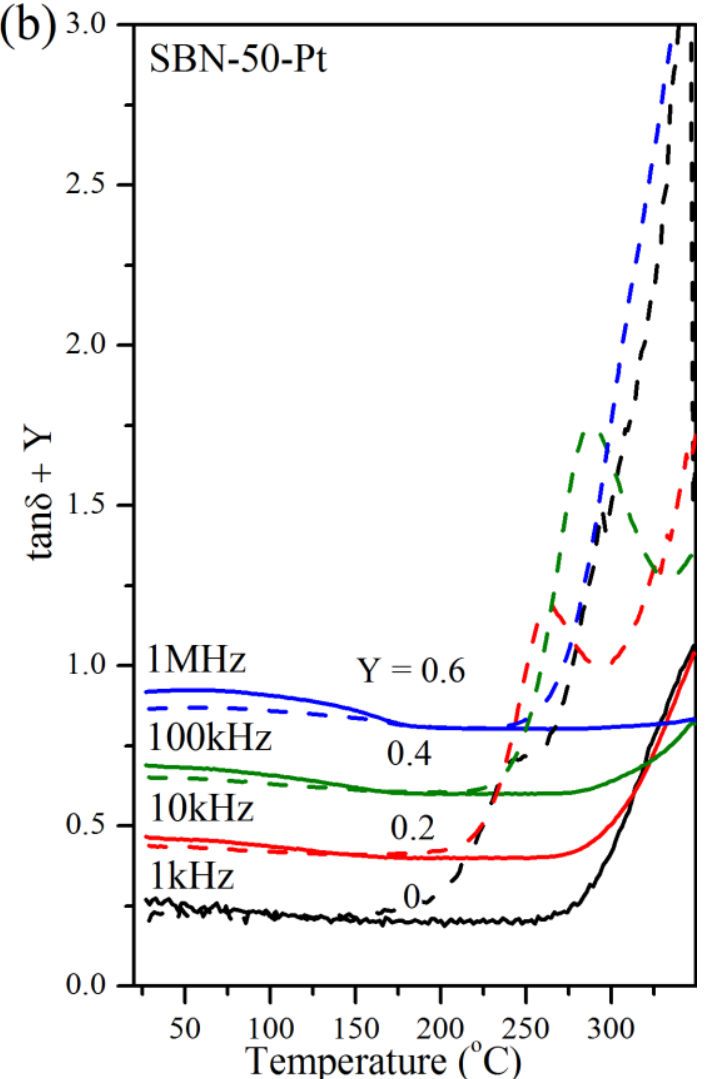

Fig. S6 The temperature dependence of (a) dielectric constant $\left(\varepsilon_{\mathrm{r}}\right)$ and (b) dielectric loss $(\tan \delta)$ at 1 $\mathrm{kHz}, 10 \mathrm{kHz}, 100 \mathrm{kHz}, 1 \mathrm{MHz}$ of SBN-50-Pt. (solid line: As-grown sample; dashed line: after poled with $\mathrm{E}=30 \mathrm{kV} / \mathrm{cm}$. The dielectric constants and dielectric loss were modified by adding certain increments (Y) to well reflect the influence of the poling effect. Insert in (a): the unmodified dielectric constant.

An abnormal increment of dielectric constant is shown in Fig. S6. The onset temperature is different with various frequencies: $210^{\circ} \mathrm{C}(1 \mathrm{kHz}), 230^{\circ} \mathrm{C}(10 \mathrm{kHz}), 250^{\circ} \mathrm{C}(100 \mathrm{kHz}), 300^{\circ} \mathrm{C}(1 \mathrm{MHz})$. Accordingly, the dielectric losses $(\tan \delta)$ also increase rapidly on the corresponding temperature by poling. The similar abnormal increment is also observed in poled SBN-50-Ag.

Oxygen vacancies exist widely in the high-temperature calcined oxides and have a great impact on the ferroelectric behaviors, such as hopping conduction. From the literature, it has been shown that with increasing temperature, an abnormally rapid increase of $\varepsilon$ and tan $\delta$ in $\mathrm{Bi}_{\mathrm{SrTiO}} \mathrm{Sr}_{3}$ happened from $220^{\circ} \mathrm{C}$ to $400^{\circ} \mathrm{C}$ and was attibuted to the short-range motion of the doubly oxygen vacanices. These dielectric behaviors resemble that of SBN-Pt pellet after poling. Thus, we attribute the abnormal increment of $\varepsilon$ and $\tan \delta$ to the production of doubly ionized oxygen vacancies due to the high electric field. Two kinds of 
oxygen vacancies are usually formed: the singly ionized one and the douly one. The former has the ionization energy of $0.1 \mathrm{eV}$, while the latter has the ionization energy which is much larger $(1.4 \mathrm{eV})$. An electrode reaction at the anode may happen under the high electric field strength, and consequently result in the production of doubly ionized oxygen vacancies.

Inevitably, the space charge region formed by the inhomogeneous distribution of oxygen vacancies maybe the origin of the leaky P-E loop. This process induces the saturated $\mathrm{V}_{\mathrm{oc}}$ on high electric field poling (ca. $30 \mathrm{kV} / \mathrm{cm}$ ), as shown in Fig. S7.

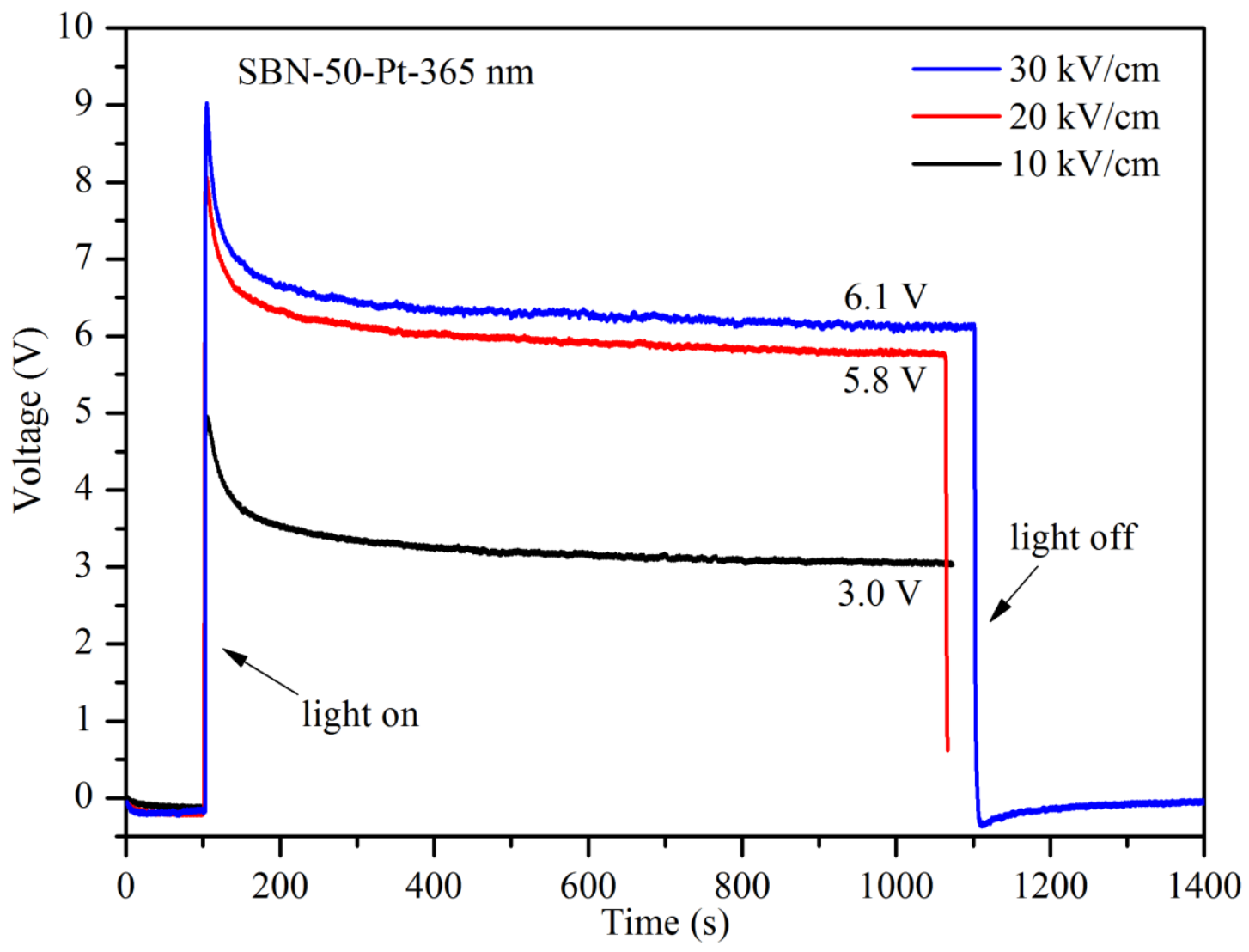

Fig. S7 Ferroelectric photovoltaic effect of SBN-50-Pt pellet poled with different electrical fields under open circuit conditions, excited with $365 \mathrm{~nm}$ LED light source. 


\section{Photoelectrochemical test for flat-band position calculation}

The flat-band position $\left(\phi_{\mathrm{fb}}\right)$ of unpoled SBN-50 can be inferred from Mott-Schottky relation:

$$
\frac{1}{\mathrm{C}_{\mathrm{sc}}^{2}}=\frac{2}{\mathrm{~N}_{\mathrm{d}} \mathrm{e} \varepsilon_{0} \varepsilon_{\mathrm{r}}}\left(\phi-\phi_{\mathrm{fb}}-\frac{\mathrm{k}_{\mathrm{b}} \mathrm{T}}{\mathrm{e}}\right)
$$

which is equal to the intercept of the M-S plots (Figure 4a). The flat band $\phi(\mathrm{NHE})$ can be converted to $\phi(\mathrm{AVS})$ from the relationship:

$$
\phi(\mathrm{AVS})=\phi(\mathrm{NHE})+0.0591 \times \mathrm{pH}+4.44(\mathrm{eV}) \text { at } 25^{\circ} \mathrm{C}
$$

(a)

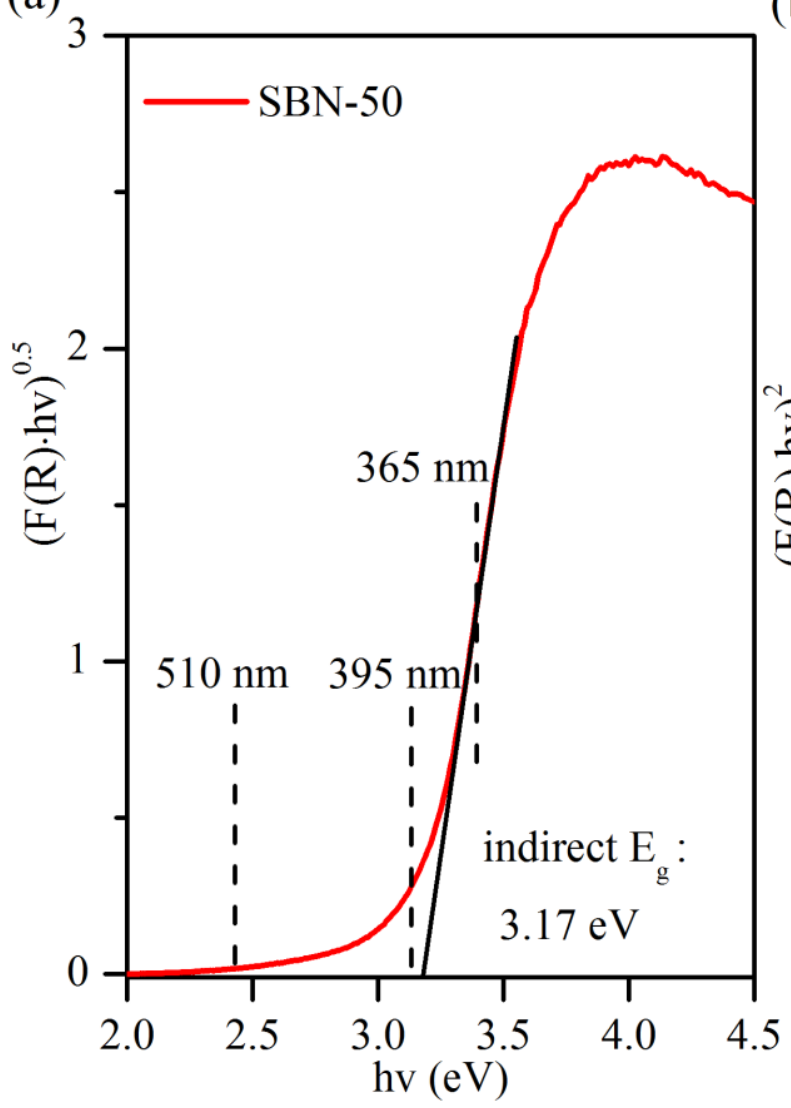

(b)

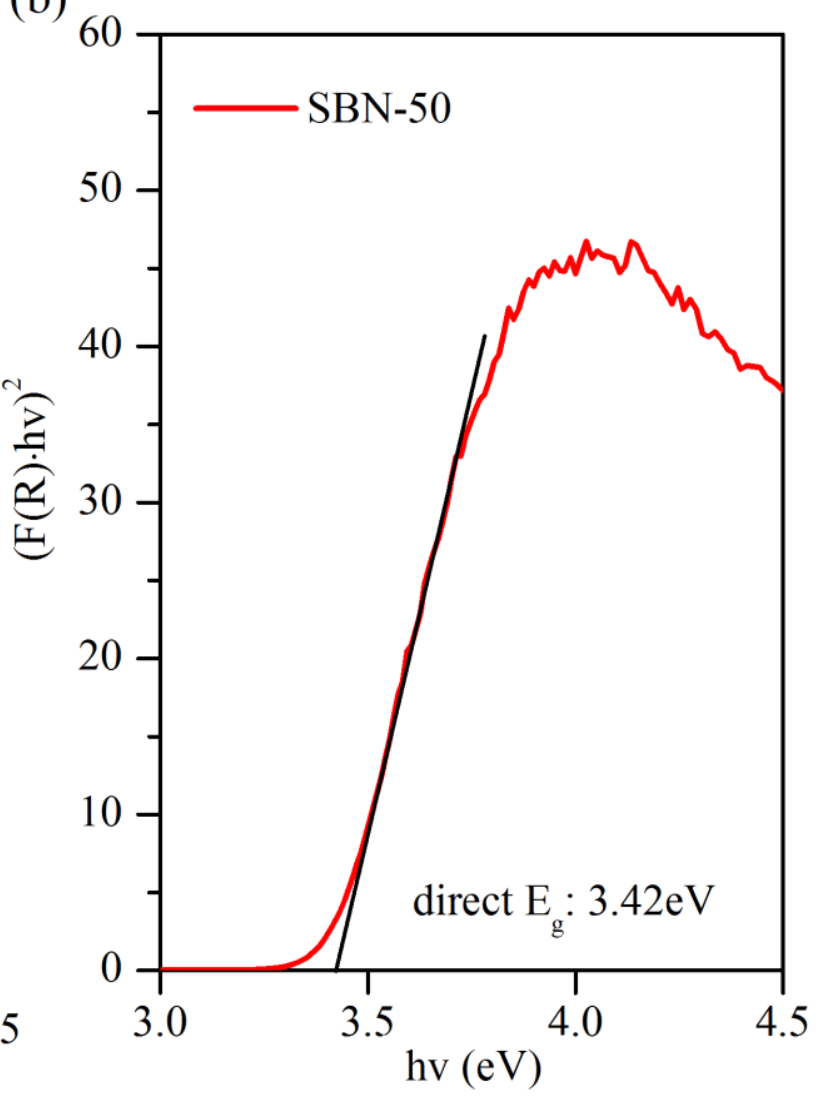

Fig. S8 Tauc plots of SBN-50-SSR powder: (a) indirect and (b) direct band gap analysis. 


\section{Photocatalysis characterization}

SBN-50 is in ferroelectric phase at RT and its $\mathrm{H}_{2}$-evolving photocatalysis activity was very low (30 $\mu \mathrm{mol} / \mathrm{g}$ for 2 hours) even with methanol as the electron donor. After the Pt loading (1wt\%) as the cocatalyst, a strongly enhanced activity was detected $(416 \mu \mathrm{mol} / \mathrm{g}$ for $2 \mathrm{~h})$.

(a)

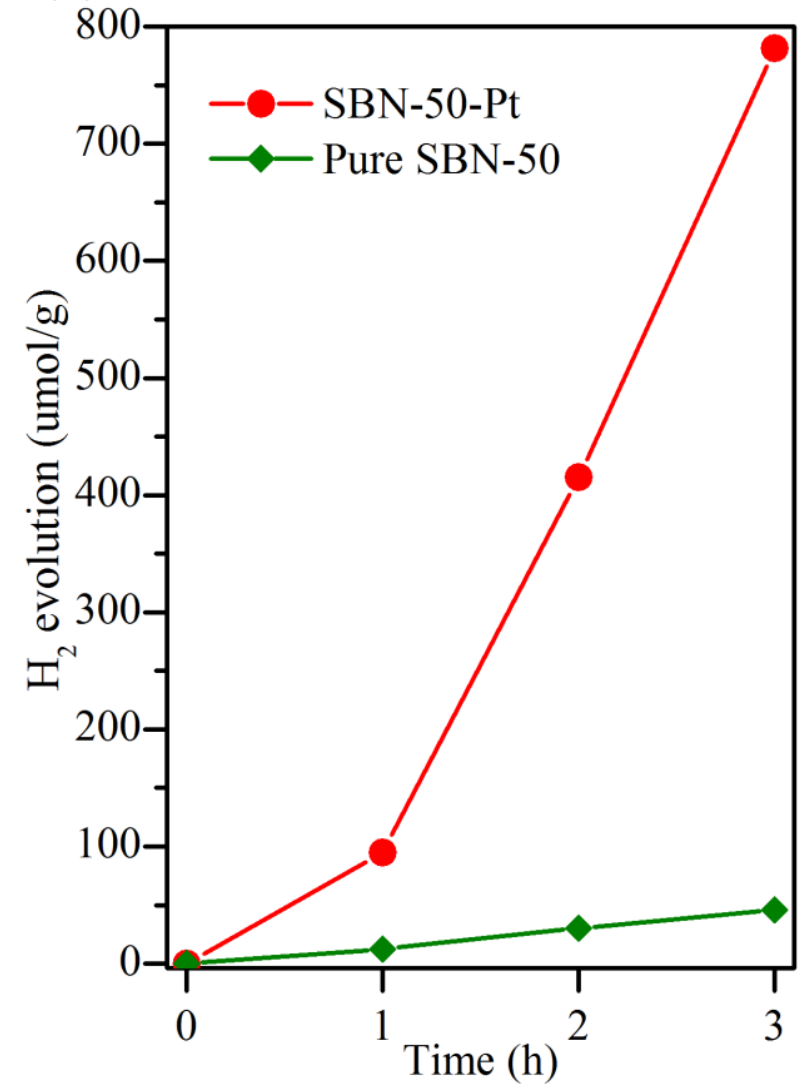

(b)

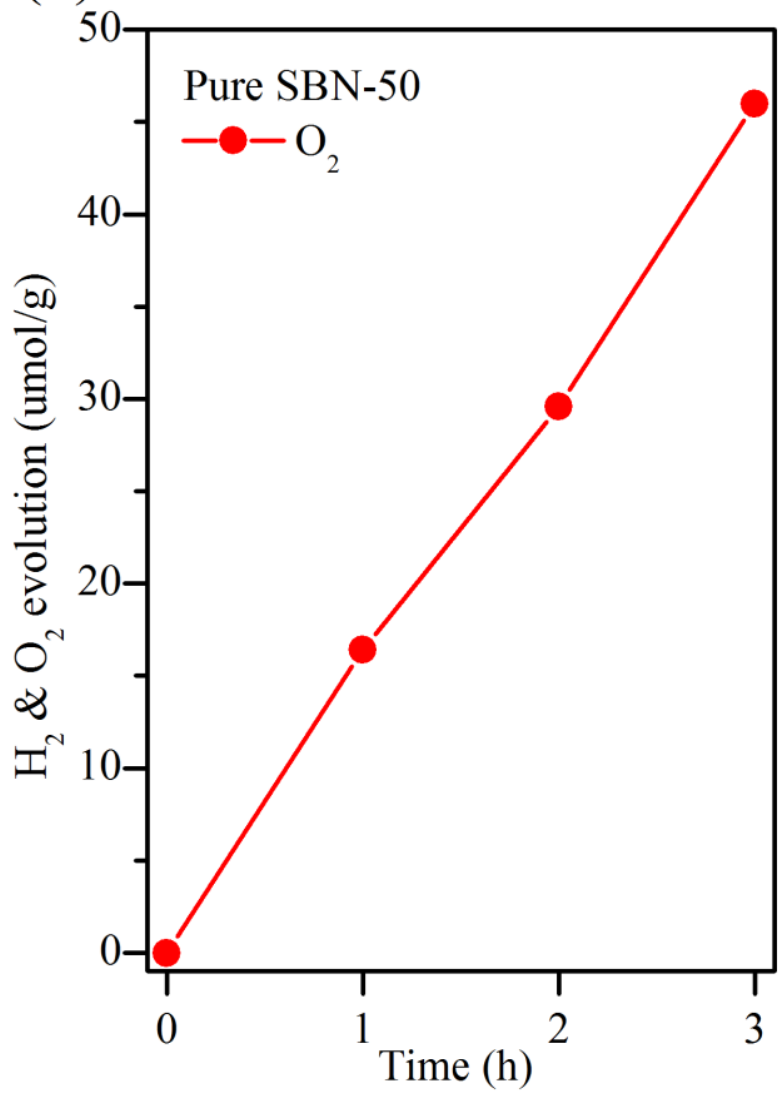

Fig. S9 (a) Reaction conditions: 0.05 g photocatalyst loaded with $1.0 \mathrm{wt} \% \mathrm{Pt}$ cocatalyst by in-situ photodeposition method; aqueous $10 \mathrm{vol} \% \mathrm{CH}_{3} \mathrm{OH}$ solution $(100 \mathrm{~mL})$; quartz top-irradiation type; 300 W Xe lamp (Cermax:PE300BUV); (b) $\mathrm{O}_{2}$ evolution from aqueous $10 \mathrm{mM} \mathrm{FeCl}_{3}$ solution (100 mL) 


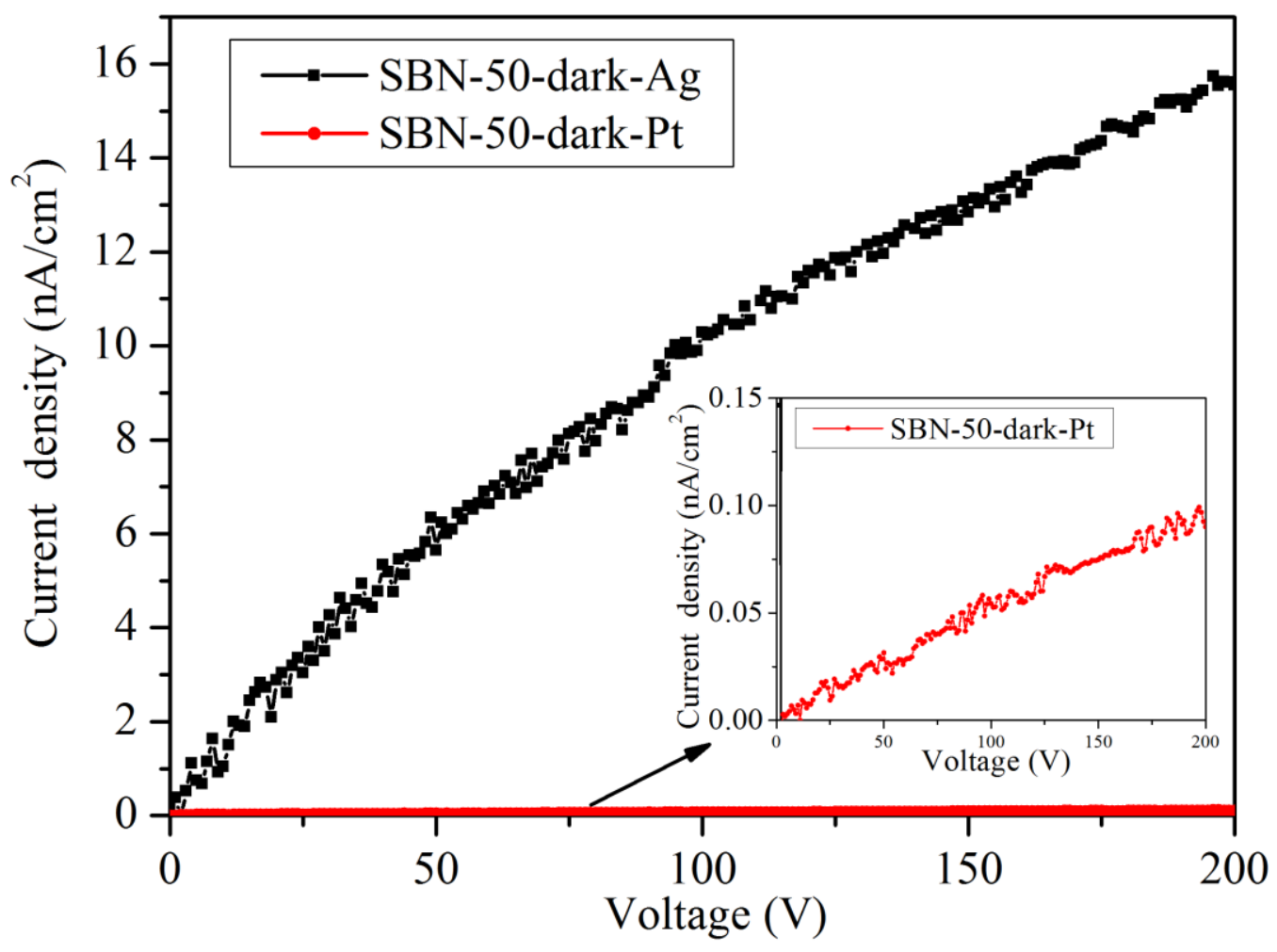

Fig. S10 I-V curves of SBN-Pt and SBN-Ag in dark

\section{Debye screening length calculation and the SCR width of Schottky barriers $\left(\mathrm{w}_{0}\right)$}

From the slope of the $1 / \mathrm{C}_{\mathrm{sc}}^{2}$ vs. $\mathrm{E}$ in Fig 4 , the positive values indicate that the majority carrier of $\mathrm{SBN}-50$ is electron, which is consistent with the fact that only the photo-oxidation reaction on the SBN-50 electrode was observed, and $\mathrm{N}_{\mathrm{d}}$ was estimated from

$$
\mathrm{K}=\frac{2}{\mathrm{~N}_{\mathrm{d}} \mathrm{e} \varepsilon_{\mathrm{o}} \varepsilon_{\mathrm{r}}}
$$

to be $4.51 \times 10^{19} \mathrm{~cm}^{-3}$. ( The relative permittivity $\varepsilon_{\mathrm{r}}(1000 \mathrm{~Hz}, \mathrm{RT})=522.2$ is from $1 / \mathrm{C}_{\mathrm{sc}}^{2}$ curve of SBN-50 pressed pellet.)

So the Debye screening length $\left(\mathrm{L}_{\mathrm{D}}\right)$ can be estimated by:

$$
\mathrm{L}_{\mathrm{D}}=\sqrt{\frac{\mathrm{kT} \varepsilon_{\mathrm{r}} \varepsilon_{0}}{\mathrm{e}^{2} \mathrm{~N}_{\mathrm{d}}}}
$$

where $\mathrm{e}=1.6 \times 10^{-19} \mathrm{C}$. We get $\mathrm{L}_{\mathrm{D}} \approx 3.8 \mathrm{~nm}$.

The SCR width of Schottky barriers $\left(\mathrm{w}_{0}\right)$ on Pt-SBN-50 interfaces $\left(\mathrm{V}_{\mathrm{s}}=1.14 \mathrm{~V}\right)$ :

$$
\mathrm{w}_{0}=\mathrm{L}_{\mathrm{D}} \cdot \sqrt{\frac{2 \mathrm{qV}_{\mathrm{s}}}{\mathrm{kT}}} \approx 38 \mathrm{~nm}
$$




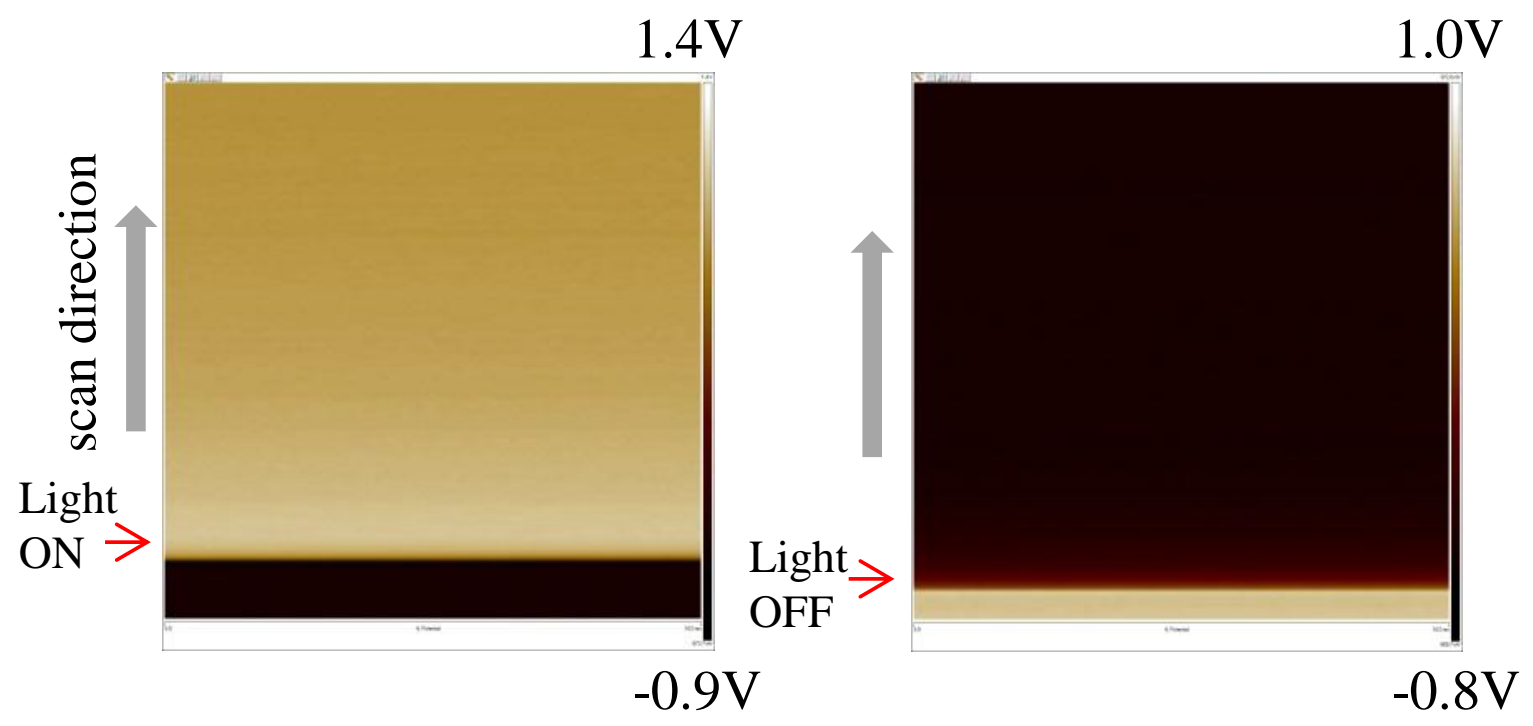

Fig. S11. KPFM images of positive poled $\mathrm{Pt}^{+}$with LED 365nm illumination after SBN-50-Pt was poled with $\mathrm{E}=30 \mathrm{kV} / \mathrm{cm}$

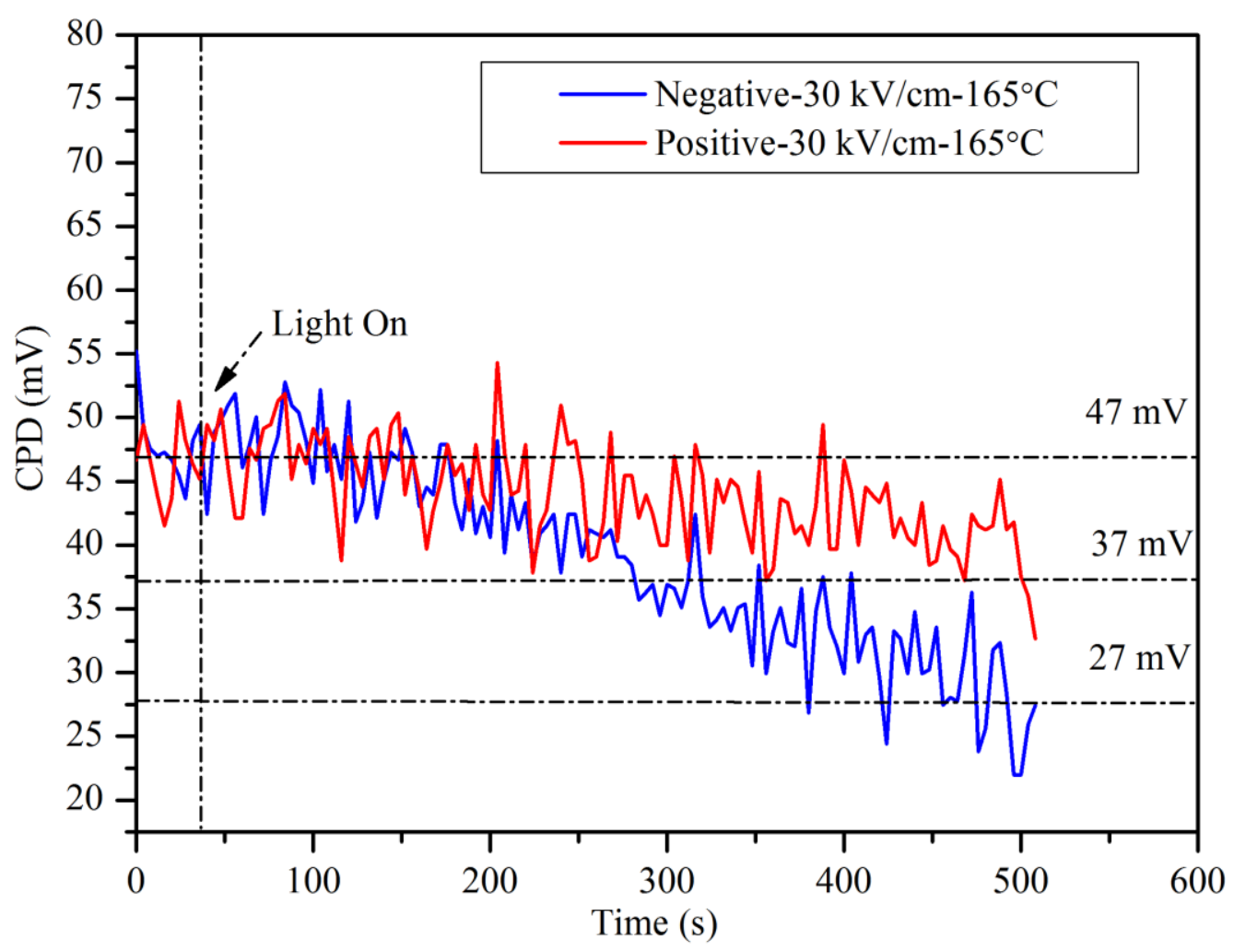

Fig. S12. The time dependent CPD profiles on two poled Ag electrodes with UV illumination on thermal depoled SBN-Ag 